\title{
A Framework of Using Customized LIDAR to Localize Robot for Nuclear Reactor Inspections
}

\author{
Dayi Zhang, Jianlin Cao, Gordon Dobie, Charles MacLeod
}

While remote inspection of industrial structures, such as nuclear reactors, using robotic crawlers currently presents significant advantages in terms of safety, accuracy and cost, other challenges emerge due to poor context-awareness and positional accuracy. This results in a lack of visibility for path planning and difficulty in precise localization of NDE (Non-Destructive Evaluation)

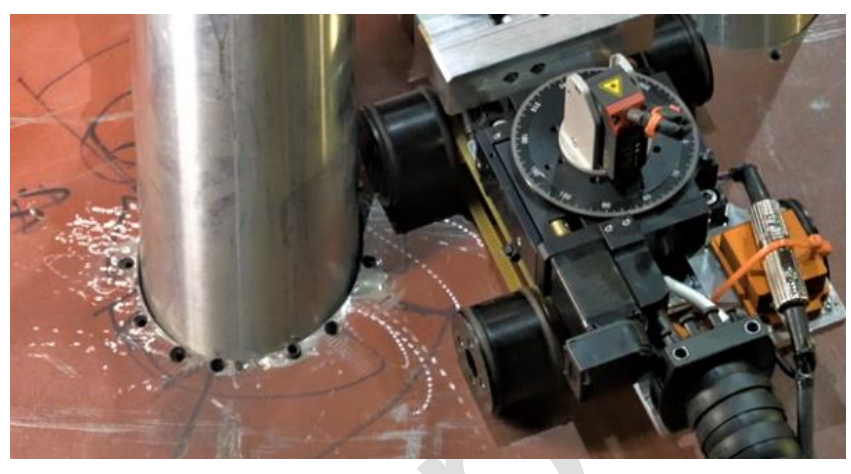
inspection data. LIDAR (Light Detection and Ranging) are one form of sensors that estimate distances at various angles to map the surrounding environment using optical techniques. Existing commercial LIDARs offer a long range of measurement, allowing mapping of the surroundings. However, such sensors often have centimeter accuracy and a minimum scan range, resulting in a blind area and are generally unsuitable for compact spaces and areas with high density of neighboring objects. This paper presents a framework for using a customized 2D laser scanner, an IMU (Inertial Measurement Unit) and a data fusion approach for localization inside high-density volumes such as nuclear reactors. The laser scanner offers precise measurements with submillimeter accuracy for items located in the short range. The IMU calculates the robot attitude angles, which are critical for inclination angle corrections. The facilities are often made of metallic materials with highly reflective surfaces, which remains problematic for the laser scanner. A mockup nuclear dome, of realistic material construction, was utilized to benchmark the performance of this framework. The distance and orientation error observed were below $2 \mathrm{~mm}$ and $1^{\circ}$, respectively. The framework will be further processed to produce a close-range environment mapping.

Keywords: Nuclear reactor inspection; Robot localization; Multiple sensor data fusion; Accuracy quantification

This work was supported in part by the Advanced Nuclear Research Centre (ANRC) in collaboration with Doosan Babcock Ltd (Renfrew, UK).

\section{Introduction}

Nuclear stations, pressure vessels and other industrial facilities are critical civil infrastructure in the modern industrialized world. Natural factors, degradations and erosion can cause damage and reduce the safe working lifetime. Such naturally occurred damage can cause other failures without regularly scheduled service [1]. Thus, periodic structural inspections are often mandated by safety regulators [2]. This body of work is focused around a pressurized water reactor, one of the most common nuclear reactors globally [3]. 
The reactor contains a closed pressure head dome and symmetrically arranged vertical rod adapters across the structure [4], [5] which limited access (the distance between two adapters $<0.5 \mathrm{~m}$ ).

Miniature, autonomous inspection robots are attractive for NDE sensor deployment and inspection applications. Such inspection robots provide flexibility to undertake inspections remotely in areas typically challenging for human access [6]-[9]. In the existing literature, crawler and drones have been utilized in various inspection tasks, including outdoor inspections like power cable integrity detections [6], wind turbine inspections [7] and in indoor inspections such as nuclear station steam generators [8] and storage casks [9].

To avoid the risk of collision and provide meaningful and sufficient inspection results, the robot operator or autonomous control algorithm needs to accurately localize the robot and the inspection payload. While satellite-based GPS (Global Positioning System) navigation systems are commonly utilized in outdoor applications, it is challenging or impossible to deploy in an indoor environment wherein satellite signals are unavailable.

Optical tracking systems are often used in indoor environments, using the distance and angle towards the known position cameras to locate objects. Such systems, include the Vicon positioning measurement systems [10], [11], offer high-accuracy optical tracking systems and a replacement for GPS when performing indoor navigation. Such systems system locate the unique geometry combination of reflective markers on an object within a tracking volume, whereby the positional accuracy of such approaches was quantified below $1 \mathrm{~mm}$ [12]. However, such systems require cameras to be fitted around the tracking volume before the inspection. The markers also need to be inside the cameras' field of view, which is not suitable for practical in-site inspections.

Monocular or stereo camera-based SLAM (Simultaneous Localization and Mapping), or visual SLAM [13], [14], is another indoor localization solution. The technique of the Visual SLAM involves extracting unique features from the real-time captured images, matching these to the previous frames and reconstructing the camera surroundings in real-time. However, real-time image processing requires enhanced competitional power, correlated to the reconstruction error [15]. The reconstruction accuracy is heavily dependent on the image quality and the number of unique features. Motion blur, camera focus, typically low indoor luminance, and fewer features impact reconstruction accuracy [7]. Radio frequency-based methods, including Radio Frequency Identification (RFID) [16], Wireless Local Area Network (WLAN) [17] and Ultra-Wide Band (UWB) [18], are also being utilized for indoor localization. The principle is using the signal flight time between beacons and mobile robots to estimate the distance between them [18]. Similar to the Vicon systems, such methods also required pre-installed beacons around the inspection volume.

Laser range finders, or LIDAR, emit a laser beam towards the obstacle and calculate the distance using the reflected beams. 2D lasers, often referred to as a laser scanner, estimates the distances at various sweep angles and provide a 2D point cloud of the measurement plane. Time-of-flight and triangulation are commonly used for laser ranging [19]. Time-of-flight utilizes the difference between transmitted and received signals to estimate the distance to the object. The triangulation technique uses the position of the 
laser spot projecting on the pixels of the camera to determine the distance [20]. Since the laser spot must reach the camera and the camera size is limited by the sensor dimensions, the triangulation sensor is more suitable for close range measurements [19]. Time-of-flight sensors are sensitive to the beam pattern integrity, which is problematic on shiny surfaces [21]. LIDAR has been utilized in various indoor SLAM applications [22]-[24], and a variety of commercial LIDAR products are available [25]. An indoor mapping system using a Hokuyo laser scanner was presented in [22], where the positional error was deemed to be smaller than $1 \%$. A similar level of accuracy was presented in [23], where the authors utilized the LIDAR to map a library corridor and in [24] a LIDAR / IMU system installed on an unmanned aerial vehicle for pipeline classifications was demonstrated with the accuracy in the range of tens of millimetres Further work in [26] demonstrated an integrated LIDAR / IMU integrated system which presented significant error reductions when using their novel ranked Kalman Filter algorithm [26].

Existing commercial LIDARs offer a long-range of measurement and geometrically mapping [27]. The sensors often have a small blind area owing to the minimum scan range [27]. It is often impractical for the applications of close-range inspections, wherein the objects are close to the robot. In addition, widespread industrial uses of metallic materials such as aluminum and stainless steels which often have a highly reflective metallic surface, which impacts the accuracy of the existing commercial LIDARs [28].

As shown in Figure 1, when considering the inspection inside a nuclear reactor, the symmetrically arranged vertical adapters [5] appear to as core obstacles but also features for robot localization. Considering the convenience of the system setup and the localization accuracy, LIDAR is the technique utilized for robot localization in this paper. However, the impact of the reflective surface and the blind spot is challenging for most commercial 2D sensors. This paper utilizes a 1D short-range laser sensor with a motorized rotation stage to build a customized 2D scanner. As shown in Figure 1, the nuclear reactor header is a dome structure, resulting in the robot being tilted during the inspection, and the laser point cloud deformed due to this inclination angle. Thus, a miniature IMU is also utilized to measure the inclination angle, reshape the point cloud and compensate for such deformations.

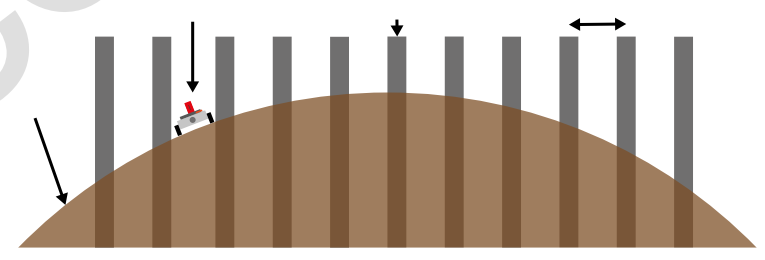

Fig. 1 A cross-section of the inspection target - a nuclear reactor header.

\section{Aim and Objectives}

This paper demonstrates a framework used for localization of inspection robots in a nuclear reactor. The inspection robot maneuvers on the closed pressure head dome and inspects the structural integrity of the reactor. The framework estimates the robot pose on the dome based on the data fusion from multiple sensors. It comprises three key components: a miniature laser distance sensor, a motorized rotation stage and an IMU, as shown in Fig. 2. 


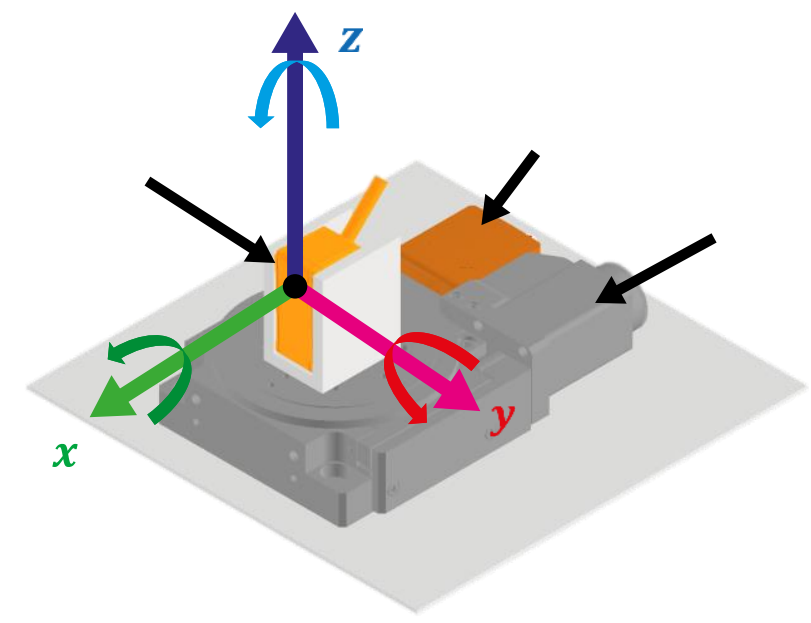

Fig. 2 Hardware setup and body reference frame used in this paper.

The miniature laser distance sensor is used to measure the distance to the surrounding environment. A Micro-Epsilon optoNCDT 1420 laser distance sensor [29] was selected primarily for its miniature dimensions (46 $\times 30 \times 20 \mathrm{~mm}$ ). The laser is a triangulation sensor with a dynamic camera exposure time for heavily reflective objects, offering better performance capability on metallic surfaces. The sensor was factory calibrated on white ceramic surfaces, with the linearity deviation below $0.2 \%$ across the measurement range (100 to $600 \mathrm{~mm}$ ). The sensor's nominal repeatability error is $0.5 \mu \mathrm{m}$, and the measuring rate is $4 \mathrm{kHz}$. The laser distance sensor was placed on a precise rotation stage [30], which spin the scanner so that the system field of view can be extended from a single point to $360^{\circ}$. The Xsens MTi-30 [31] sensors was selected primarily for its compact dimensions $(57 \times 42 \times 24 \mathrm{~mm})$. It is a precise IMU to provide the platform attitude angle, critical for the inclination angle corrections when the robot is not parallel to the ground.

Section 3 characteristics the laser scanner accuracy on the reflective surface with different tilt angles, while Section 4 presents the methodology and algorithms used for position estimation and localization. Section 5 evaluates the accuracy of the pose estimated by the framework and finally, in Section 6 , the paper is concluded with a discussion of the results.

The main contributions of this paper are:

- Development of a framework that can be used for robot inspection of a nuclear reactor. The framework is based on the data fusion from a miniature 1D laser, a precision rotation stage, an IMU.

- Evaluation of a laser scanner performances on metallic surfaces.

\section{Laser Accuracy Characterization}

The laser sensor is critical to the positioning system because it underpins the accuracy of the entire system. The following experiment is designed to characterize the laser measurement accuracy. 
The laser was mounted on the end of a KUKA KR6: an industrial robotic manipulator available in our laboratory. Utilizing the internal pose feedback, the robot pitch, roll and yaw angles and $(x, y, z)$ translations, measured with $0.01^{\circ}$ angular and $0.01 \mathrm{~mm}$ translational resolution, respectively, were manually adjusted to move the laser through a range of poses. The manipulator has sub-millimeter accuracy and a repeatability of $0.03 \mathrm{~mm}$ [32]. The KUKA manipulator deploys the laser scanner to more precise positions, granting the experiment repeatability and providing more accurate results. The experiment comprises three evaluation scenarios: standoff test, pitch angle test and yaw angle test, illustrated in Fig. 3.

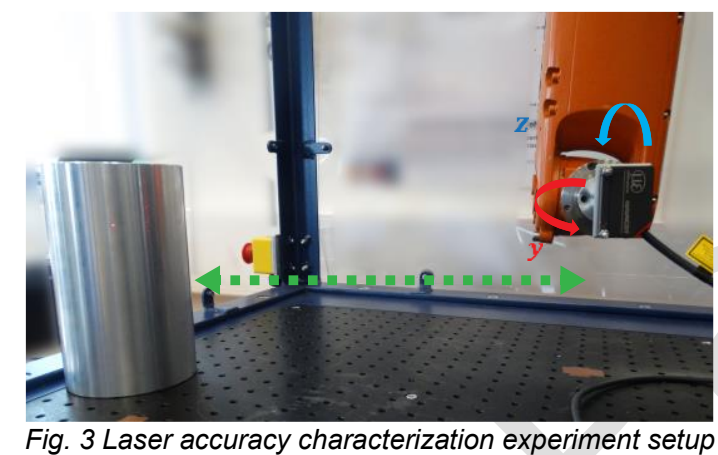

The laser sensor was perpendicular to the aluminum sample surface, while the pitch, roll and yaw angle are $0^{\circ}$. The manipulator moved the laser sensor from $100 \mathrm{~mm}$ to $600 \mathrm{~mm}$ standoff, with $100 \mathrm{~mm}$ increment. The pitch angle experiment presented the situation while the platform is tilted on the dome. The yaw angle experiment demonstrated the sensor accuracy when it was rotated for a complete planar scan. In these two tests, the laser sensor was at the middle of its measurement range (350 mm standoff to the sample surface), and the manipulator tilts the laser pitch and yaw angle to the maximum degree that the sensor gets reflections, with $1^{\circ}$ increment.

Fig. 4 shows the experiments results from the standoff test, pitch angle test and yaw angle test, respectively.

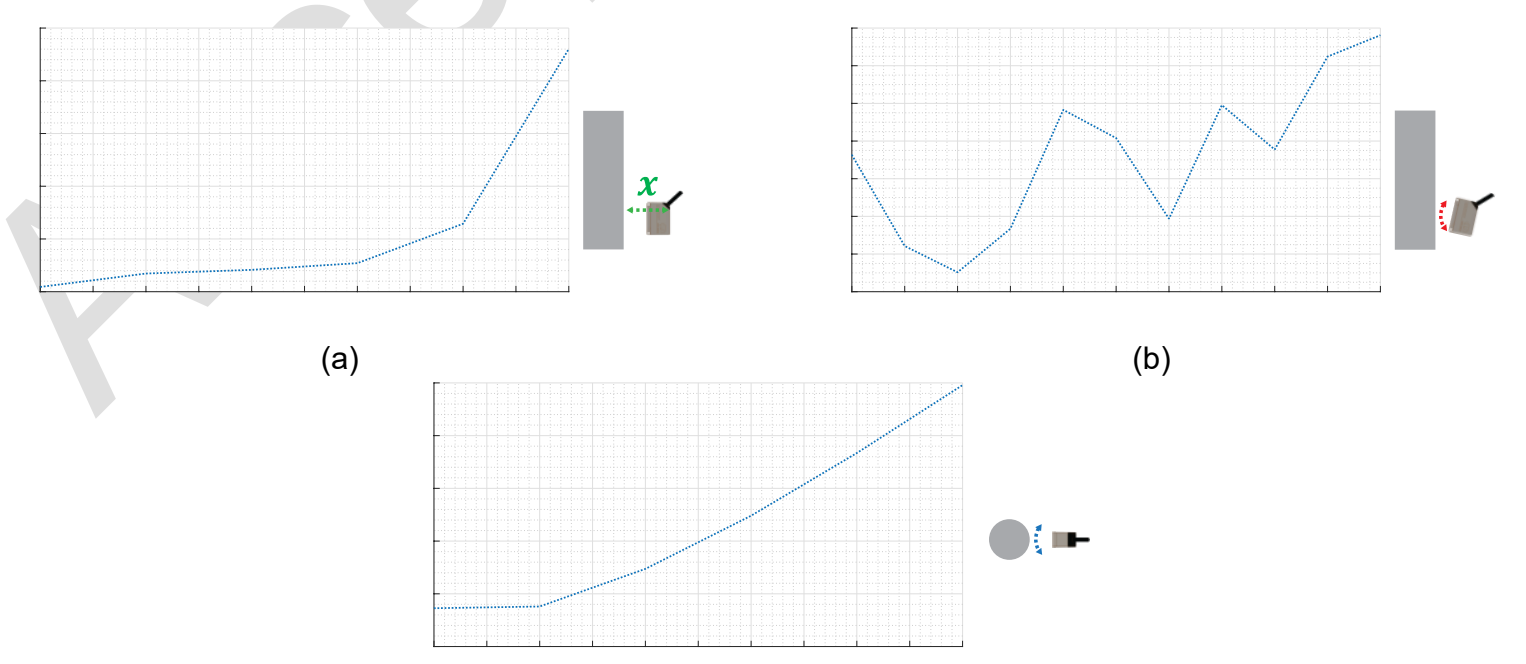

(c) 
Fig. 4 Range Measurement error when (a) varying the laser standoff. The error while the laser was at the middle of its range (350 $\mathrm{mm}$ ) while the laser was (b) tilted the laser pitch angle (c) tilted the laser yaw angle.

As shown in Fig. 4(a), the error increases with the increment of the standoff distance. It was reached 2.3 $\mathrm{mm}$ while the standoff is $600 \mathrm{~mm}$. Fig. 4(b) shows the majority of the errors when tilting the sensor pitch angle were below $1 \mathrm{~mm}$. Fig. 4(c) shows the error introduced by the yaw angle is higher than those introduced by the standoff and pitch angle. A $10 \mathrm{~mm}$ error was observed while the laser was rotated $7^{\circ}$ along the Z-axis.

Such errors are due to the optical characteristic of the laser sensor. When the laser angle is tilted, the incidence angle is non-zero. The main component of the incident beam is reflected to other directions. Less energy was reflected into the direction of the laser receiver [21]. Fig. 5 and Fig. 6 show the laser dot on the pipe and the camera sensor reading while the sensor yaw angle is tilted. When compared with Fig. 6(b), it is difficult for the sensor to identify the laser reflection from Fig. 6(a). The received energy was $30 \%$ of the power received at $0^{\circ}$ yaw angle.

Fig. 5 Laser dots on the aluminum sample when the laser yaw angle was tilted. The laser dot lost its integrity and was spread as a line.

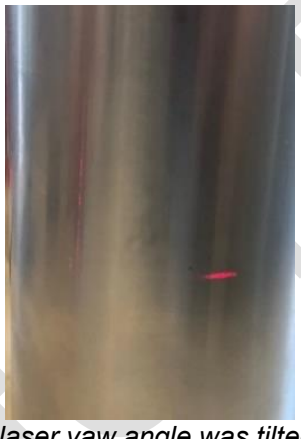

(a)

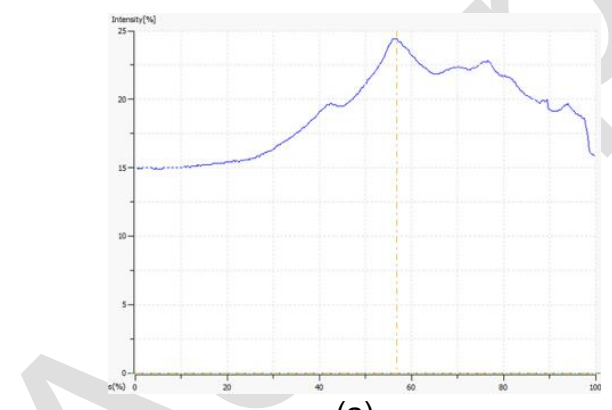

Fig. 6 Screenshot of the laser receiver readings (a) Relatively low SNR when the yaw angle was tilted. This is due to the laser dot spread on the aluminum surface leading to the measurement error (b) Good SNR when the yaw angle was $0^{\circ}$.

\section{Lab Mock-up Test Bench}

8 metal cylinders of $110 \mathrm{~mm}$ diameter were fabricated and set up in the laboratory to mimic an inspection scenario, featuring vertical rod adapters to mock a nuclear reactor vessel [4], [5] as presented in Fig. 7(a). Dimensions of the cylinders are defined to provide a suitable scale to replicate inspection conditions in proximity to nuclear header structures provided by the nuclear station operator. The Vicon photogrammetry positioning system, was used as a ground truth senor for this accuracy validation and documented sub- 
millimeter accuracy [10], [11], comprising twelve optical cameras and real-time tracking the translations and orientations of the object at $100 \mathrm{~Hz}$. As shown in Figure 7(b), six asymmetrical Vicon markers were placed for Vicon positioning tracking. The pattern of these markers locates the position of the center of the system as well as the center of each component (laser, IMU, rotation stage). The center of the Vicon tracking object matches the center of the laser scanner, which is the system's origin. Additionally, a graph paper with a $5 \mathrm{~mm}$ grid width was placed under the mock-up to help visual training and pose tracking.
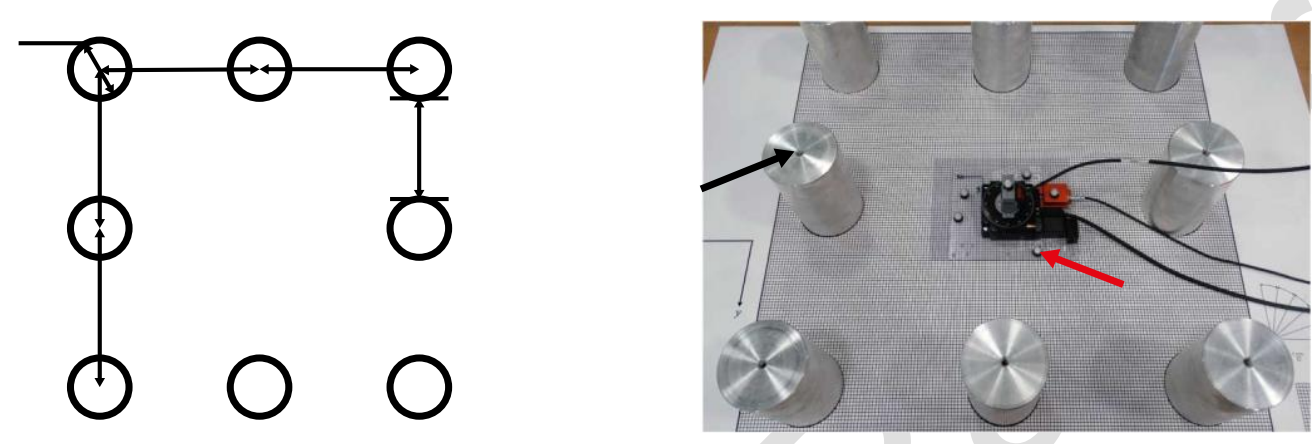

Fig. 7 (a) The lab mock-up schematic diagram. (b)The lab mock-up to simulate the actual inspection scenario on the nuclear reactor.

\section{Localization Algorithm}

Matching 2D LIDAR scan is the fundamental component of the localization algorithm which require finding the correspondence between the features of two LIDAR scans and converting the correspondence to the actual pose transform. Several advanced matching algorithms were proposed to minimize the error. Cox [33] used points as primitives and matched them to lines given in a priori model. The algorithm is designed for use in a structured office or factory environment rather than unstructured natural environments. The matching algorithms proposed in [34], [35] extracted lines from the scans to match with the models. Similar to [33] method, this algorithm was able to only resolve office-like environments with matt surfaces, as well as with environments without orthogonal and rectilinear walls. However, the environment herein is filled with circular pipes and highly symmetric, which is not suitable for such a schem. EKF-SLAM [36], FastSLAM [37] and other SLAM-based algorithms fuse LIDAR point clouds and IMU motions for robot indoor navigations and mappings. Considering, within this body of work, the IMU is proposed to correct the inclination angle, less geometrical features and more computational requirements, SLAM-based algorithms are not fitting herein.

Matching points to points [38] is another approach that has serials of variants called ICP (iterated closest point [39]-[41]). Initial guesses, pre-processing such as triangulation, mesh simplification is compulsory in such an algorithm. Compared with the algorithm implemented herein, it is relatively slow due to the time consumption for points pairing. Moreover, such an algorithm is relatively sensitive to laser accuracy. It requires a highly accurate measurement sensor to optimize the pairing precision. 
The Normal Distribution Transform [42] is the algorithm utilized herein for point cloud matching. The algorithm is selected due to its speed and accuracy [43]. The principle is converting the current scan into a binary grid map and projecting the reference map on it to estimate the travel pose, as shown in Fig. 8.

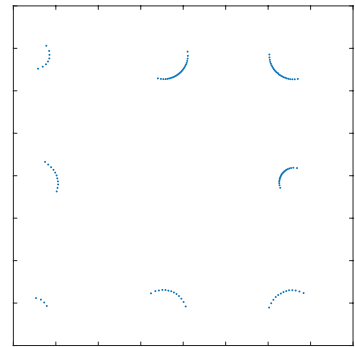

(a)

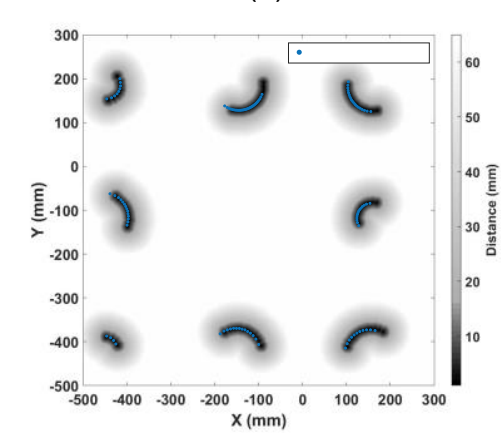

(c)

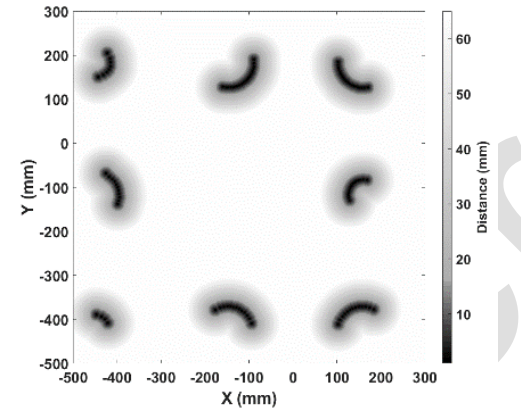

(b)

Fig. 8 Pose estimation process. The raw laser point cloud (a) is firstly transfer to a binary grid map (b). Then the point cloud captured previous is projected on the binary grid map (c). (d) is a section from the previous laser scan projected on the binary grid map

Firstly, the LIDAR captures a raw $360^{\circ}$ mapping (Fig. 8(a)), which is then transferred to a grid map. The color density of each grid represents the Euclidean distance to the closet laser point (Fig. 8(b)). Then, the reference map (point cloud from previous scans) is projected on the grid map (Fig. 8(c)). Each point on the reference map can be geometrically correlated to a grid on the binary map. Since the grid values are the Euclidean distance to its nearest point from the new scan, each correlation represents the distance $\left(d_{n}\right)$ between the reference map and the new scan, shown in Fig. 8(d).

By moving and rotating the reference map, the distances between the map and the new scan $\left(d_{n}\right)$ are varying. The new scan and the reference map can be considered as geometrically matched when the sum of these distance is minimized, defined as:

$$
\mathrm{J}=\sum_{m=0}^{n} d_{m}
$$

The algorithm output is the translational and oriental transform between the reference map and the current new scan. Because the environment is symmetrical, the difference in the distance and orientation between two scans are limited to under $\pm d / 2$ and $\pm 45^{\circ}$, where $d$ is the distance between two symmetric nuclear adapters. 


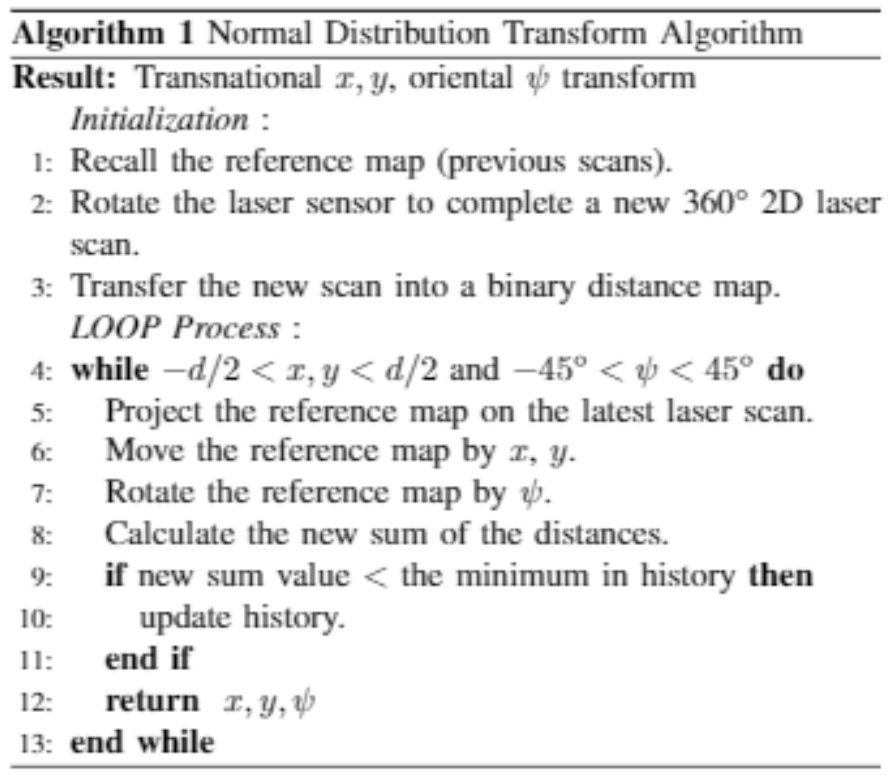

The conventional normal distribution transform as Eq. 1 minimizes the average distance between the two measurements. Depending on the laser scanner position, vertical adapters near the laser naturally has more valid data points than adapters in the distance. The point cloud density is varying depending on the distance to the laser scanner. The closet vertical rod adapter has the highest density points, while the furthest has the sparse points. It is possible a small quantity of large matching errors from the distant adapters being averaged with many small errors from the neighbors, causing a biased result. Therefore, a secondary optimization is utilized to mitigate such uneven density issues after the conventional pose estimation.

Instead of averaging errors across all points, the optimization utilizes an averaged error from each adapter. First, the algorithm identifies and groups the adapters from the point cloud. Then, it calculates the matching errors of each adapter. Subsequently, the errors from each adapter are summed to be an overall error. Each adapter is having similar contributions to this overall error. Then, like Algorithm 1 the optimization is to minimize the new sum of the error. Since the optimization is expected to find a local minimum with a relatively small adjustment and optimize the processing time, the translational and oriental searching margins are reduced to $\pm 2 \mathrm{~mm}$ and $\pm 2^{\circ}$.

The point clouds from the previous and latest measurements are plotted together with $(x, y, y a w)$ on the bespoke interface software, as shown in Fig. 9. 


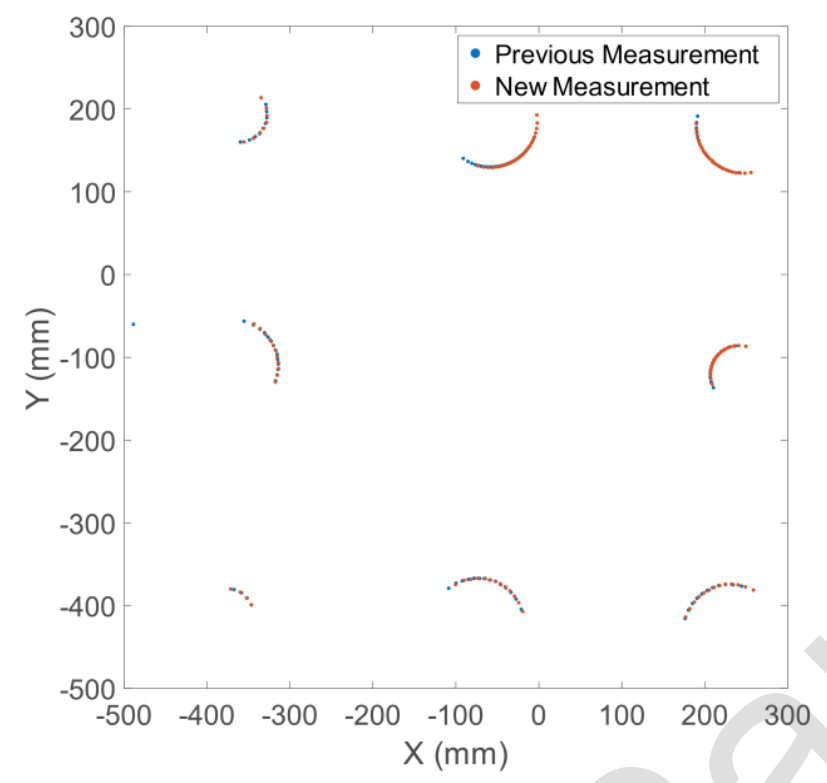

Fig. 9 The new laser measurement stitches on the previous measurement. The point clouds are perfectly matched.

The robot is not parallel to the ground while inside the nuclear reactor. The point cloud is deformed under this condition. Thus, the onboard IMU is utilized to compensate for such deformation. The inclination angle correction is based on the pitch and roll readings from the IMU. The platform base, including the IMU, is stationary during the laser scanning. The IMU readings during the laser measurement period are averaged 360 to reduce the noise. Then, the averaged values synchronized to the laser data and utilized for angle compensation. The output from the matching algorithm is the $x, y$ and yaw movements in the manipulator local coordinate. The poses are then transferred to the global coordinate by:

$$
\begin{gathered}
\psi_{t}=\psi_{t-1}+\psi_{t} \\
{\left[\begin{array}{l}
x_{t} \\
y_{t}
\end{array}\right]=\left[\begin{array}{l}
x_{t-1} \\
y_{t-1}
\end{array}\right]+\left[\begin{array}{cc}
\cos \left(\psi_{t}\right) & -\sin \left(\psi_{t}\right) \\
\sin \left(\psi_{t}\right) & \cos \left(\psi_{t}\right)
\end{array}\right]\left[\begin{array}{l}
x_{t} \\
y_{t}
\end{array}\right]}
\end{gathered}
$$

\section{Accuracy Evaluation}

This validation experiment includes three tilt angles $\left(0^{\circ}, 15^{\circ}, 30^{\circ}\right)$ to simulate the robot poses on the dome, as shown in Figure 1.

Each tilt angle is tested in two specific scenarios. The first scenario is keeping the yaw angle as $0^{\circ}$, while the second one rotates the yaw angle during the measurement, as shown in Fig. 10. The first demonstrates the accuracy while the crawler is well controlled to follow the desired trajectory. The second one presents the accuracy while the crawler does not strictly follow the trajectory. The trajectory comprises nine fixed waypoints with fixed distances to the centers of cylinders, which mimic robot parking positions while conducting inspections in a $500 \times 500 \mathrm{~mm}$ region. The measurement starts from waypoint 1 , undertakes a pose measurement and travels to the next point. The platform is aligned with the Vicon and the graph paper and ensure the experiments starting from the same pose and initial state. The localization results 
are compared to the global positions given by the Vicon system with the sub-millimeter accuracy. All measurements have been repeated three times independently to provide key repeatability information. Table 1 listed the results of the validation experiments.

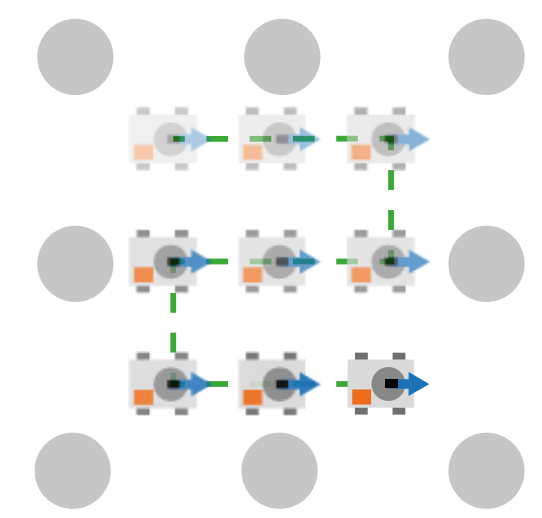

(a)

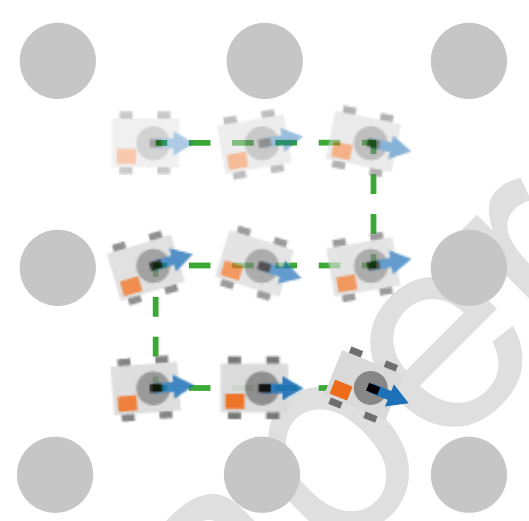

(b)

Fig. 10 The trajectory while undertaking the pose accuracy validation. The blue arrow represents the robot heading direction. (a) is when the yaw angle was constant as $0^{\circ}$ (b) is when the yaw angle was varying. The system started from waypoint \#1 and finished at waypoint \#9. The color of the robot stands for the experiment time. Brightest means the start (waypoint \#1).

Table 1 Validation experiment results

\begin{tabular}{|l|l|l|l|l|l|l|}
\hline \multirow{2}{*}{} & \multicolumn{2}{|c|}{$0^{\circ}$ Tilt Angle } & \multicolumn{2}{c|}{$15^{\circ}$ Tilt Angle } & \multicolumn{2}{c|}{$30^{\circ}$ Tilt Angle } \\
\cline { 2 - 7 } & $\begin{array}{l}\text { Constant } \\
\text { Yaw }\end{array}$ & $\begin{array}{l}\text { Varying } \\
\text { Yaw }\end{array}$ & $\begin{array}{l}\text { Constant } \\
\text { Yaw }\end{array}$ & $\begin{array}{l}\text { Varying } \\
\text { Yaw }\end{array}$ & $\begin{array}{l}\text { Constant } \\
\text { Yaw }\end{array}$ & $\begin{array}{l}\text { Varying } \\
\text { Yaw }\end{array}$ \\
\hline $\begin{array}{l}\text { Mean Distance } \\
\text { Error (mm) }\end{array}$ & 0.551 & 0.552 & 0.557 & 0.578 & 1.445 & 1.265 \\
\hline $\begin{array}{l}\text { Stand Deviation } \\
\text { Distance Error } \\
(\mathrm{mm})\end{array}$ & 0.244 & 0.287 & 0.292 & 0.346 & 1.026 & 0.704 \\
\hline $\begin{array}{l}\text { Mean Yaw Error } \\
\left({ }^{\circ}\right)\end{array}$ & 0.346 & 0.235 & 0.153 & 0.297 & 0.643 & 0.429 \\
\hline $\begin{array}{l}\text { Stand Deviation } \\
\text { Yaw Error }\left(^{\circ}\right)\end{array}$ & 0.091 & 0.117 & 0.132 & 0.270 & 0.351 & 0.279 \\
\hline
\end{tabular}

As shown in Table 1, the errors of the (x,y) position were below $2 \mathrm{~mm}$, and the yaw angle errors were below $1^{\circ}$. The sources of these positioning errors are raw laser measurement error (as presented in Section 3), IMU measurement errors and the scanning resolution.

The matching errors, including the $(\mathrm{x}, \mathrm{y})$ position and the yaw angle, were significantly increased with the incrementation of the system tilting angle. Compared with the results from $0^{\circ}$ angle, the translational and orientational accuracy from $30^{\circ}$ tilting angle were degraded by a factor of 3 and 2 . Such degradations can be the result of the range measurement errors when the sensor was tilted. Moreover, the IMU is specified as having a maximum of $0.2^{\circ}$ error, which can cause a $2.1 \mathrm{~mm}$ translational error at the distance of 600 $\mathrm{mm}$.

The scanning resolution denotes the laser points' density for pose matching, impacting the measurement accuracy [23]. Increasing the density of laser points can improve the accuracy as well as the time 
consumption for each measurement. Herein the scan is the laser measurement per degree, which is optimized for time consumption and measurement accuracy.

\section{Conclusion}

This paper presents a new framework to localize robotic crawlers in heavily dense and congested environments such as nuclear reactors. Using the combination of a 1D laser scanner and the IMU data, the system successfully estimates the pose in the simulated complex environment. The paper evaluated laser scanner accuracies on highly reflective steel surfaces, typically present on industrial facilities. Using this framework, the mean error of the translational and orientation pose during the experiment were below 2 $\mathrm{mm}$ and $1^{\circ}$ on the $600 \times 600 \mathrm{~mm}$ mockup, respectively. Thus, the framework proposed in this paper can be used to estimate and localize the robot position during inspections of such reactors. It is worth noting that the framework is not only limited to use for this specific inspection target. It can also be applied to other inspection scenarios where the environment is crowded and contains many metallic features. Finally, it is worth noting that, the IMU in this system is utilized for laser point cloud angle corrections. Therefore, it only takes measurements while the robot is stationary. However, the IMU can be used to measure motions while the robot is moving. This constitutes an opportunity to further research upon the optimizations of the position accuracy with SLAM-based algorithms and point cloud optimizations [36], [44]. Future work will consider further integration of other onboard sensors, such as wheel encoders, to further reduce the positional error.

\section{Reference}

Rob. Auton. Syst., vol. 75, pp. 490-507, 2016.

[2] "O $-0$ G [Online]. Available: https://www.hse.gov.uk/foi/internalops/og/og-00037.htm. desalination: A state-of-the- $\quad$ v $\quad$ "Desalination, vol. 457, pp. 39-61, May 2019.

Encyclopedia of Energy, Elsevier, 2004, pp. 333-340.

[6]

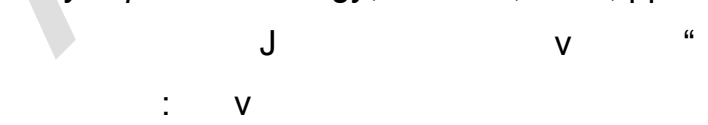

$\checkmark \quad$-based power line

"Int. J. Electr. Power

Energy Syst., vol. 99, pp. 107-120, 2018.

G
C

K G v "Eng. Struct., vol. 209, p. 109940, Apr. 2020. 
steam generator secondary side with self- $\quad z \quad$ "Robot. Biomimetics, vol. 4, no. 1, p. 26, Dec. 2017.

[9] C. J. Lissenden et al. "T

C

J. Press. Vessel Technol., vol. 139, no. 3, Jun. 2017.

[10]

"G F

T-

https://www.viconpeak.com/downloads/documentation/go-further-with-vicon-mx-t-series.

[Accessed: 01-Aug-2019].

[11]

P. Merriaux, Y. Dupu

B

V “

V

"Sensors (Switzerland), vol. 17, no. 7, 2017.

[12]

R. Summan et al. “ V

Meas. J. Int. Meas. Confed., vol. 68, pp. 189-200, 2015.

[13] J $\mathrm{T}$ C “ -SLAM: Large-

Notes Comput. Sci. (including Subser. Lect. Notes Artif. Intell. Lect. Notes Bioinformatics), vol. 8690 LNCS, no. PART 2, pp. 834-849, 2014.

[14] L. R. Ramírez-Hernández et al. “ V V -dimensional point localization accuracy in stereo $\mathrm{V}$

\section{V}

"Int. J. Adv. Robot. Syst., vol. 17, no. 1, 2020.

[15] H. Strasdat, J. M. vol. 30, no. 2, pp. 65-77, Feb. 2012.

[16] E. Hatem, S. Abou-Chakra, E. Colin, J.-M. Laheurte, and B. Eland Generalization Capability of RFID Tag ' C z " Sensors, vol. 20, no. 15, p. 4100, Jul. 2020.

[17] K. Wang et al. "

$\mathrm{F}$

[18] $\quad$ Z $\quad \mathrm{T}$ v W

" IEEE Access, vol. 7, pp. 72308-72315, 2019. : W ? Image Vis. Comput., 
[24] G j $\mathrm{K} \quad \mathrm{K}$ $\mathrm{K}$ indoor navigation system for UAVs and its application in real(Switzerland), vol. 17, no. 6, 2017.

" A Rev. Point Cloud Regist. Algorithms Mob. Robot., 2015.

[26]
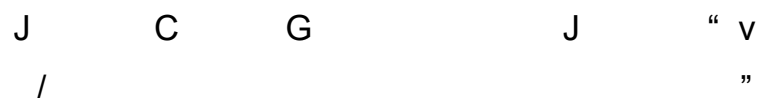

O “

C

https://autonomoustuff.com/lidar-chart.

[28] C

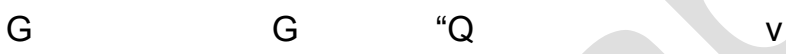

data when scanning industrial materials " IEEE Sens. J., vol. 16, no. 22, pp. 7999-8009, 2016.

[29]

Micro- " C T "O $\mathrm{O}$ : : /l

epsilon.co.uk/displacement-position-sensors/laser-sensor/optoNCDT_1420_basic/.

http://www.standa.It/products/catalog/motorised_positioners?item=245.

[Accessed: 20-Sep-2019].

[32] M. Morozov et al. “ ccuracy of industrial robots through metrology for the enhancement of automated non-

v " IEEE Int. Conf. Multisens. Fusion Integr. Intell. Syst., pp. 335-340, 2017.

J C "B -an experiment in guidance and navigation of an au IEEE Trans. Robot. Autom., vol. 7, no. 2, pp. 193-204, 1991.

J.- $G$

$\mathrm{T} W$

V
B " $\mathrm{F}$ IEEE/RSJ International Conference on Intelligent Robots and

Systems, 1999, pp. 1412-1419.

J.- $G$

Z
C $\mathrm{V}$

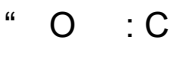

" Proceedings of the First Euromicro Workshop on

Advanced Mobile Robots (EUROBOT '96), 1996, pp. 61-67.

[36] Z W

C $\mathrm{K}$

B C “ -assisted 2D SLAM method for low-texture V

" Appl. Sci., vol. 8, no. 12, 2018.

[37] S. Thrun, M. Montemerlo, D. Koller, B. Wegbreit, J. Nieto, and E. Nebot, " $\{\mathrm{F} \quad\}:\{\quad\}$ Efficient Solution to the Simultaneous Localization and Mapping Problem with Unknown Data

" J. Mach. Learn. Res., 2004.

[38] $F$

Range S "J. Intell. Robot. Syst., vol. 18, no. 3, pp. 249-275, 1997. 
[39] J B

Mach. Intell., vol. 14, no. 1, pp. 239-256, 1992.

[40] $\quad$ C

G

"O j

$" \quad$ IEEE

International Conference on Robotics and Automation, 1991, pp. 2724-2729.

[41] Z Z " V F $\quad$-Form Curves and Surfaces " Int. J. Comput. Vis., vol. 13, no. 2, pp. 119-152, 1994.

[42] B " $\mathrm{T}$ Int. Conf. Intell. Robot. Syst., vol. 3, pp. 2743-2748, 2003.

[43] C $\quad z \quad Z \quad$ " -Time Graph-Based SLAM with Occupancy Normal Distributions

T "Proc. - IEEE Int. Conf. Robot. Autom., pp. 3106-3111, 2020.

[44] Z F Z "B : B j "IEEE Robot. Autom. Lett., vol. 6, no. 2, pp. 3184-3191, 2021. 